\title{
Feasibility of Endoscopic Transforaminal Lumbar Interbody Fusion (eTLIF) Through the Posterior Paraspinal Approach: Technical Note and Preliminary Result
}

\author{
Hyeun Sung Kim ${ }^{1}$, Harshavardhan Dilip Raorane ${ }^{1,2}$, Pang Hung $\mathrm{Wu}^{1,3}$, Dong Hwa Heo ${ }^{1}$, Yeon Jin $\mathrm{Yi}^{1}$, \\ Kyung-Hoon Yang $^{1}$, Il-Tae Jang ${ }^{1}$ \\ ${ }^{1}$ Department of Neurosurgery, Nanoori Gangnam Hospital, Seoul, Spine Surgery, Seoul, Republic of Korea \\ ${ }^{2}$ Kolhapur Institute of Orthopedics and Trauma, Kolhapur, India \\ ${ }^{3}$ Department of Orthopaedic Surgery, National University Health System, Jurong Health Campus, Singapore
}

Corresponding Author:

Hyeun Sung Kim, MD, PhD

Department of Neurosurgery, Nanoori

Hospital Gangnam, Seoul 731, Eonju-ro,

Gangnam-gu, Seoul, Republic of Korea

Tel: $+82-2-6003-9767$

Fax: +82-2-3445-9755

Email: neurospinekim@gmail.com

Received: January 31, 2021

Revised: March 22, 2021

Accepted: March 29, 2021
Objective: The implement of endoscopic spinal surgery has minimized the requirement of fusion procedures. However, certain patients still require fusion surgery such as instability. We performed a full-endoscopic transforaminal lumbar interbody fusion (eTLIF) through a conventional paraspinal approach. Methods: eighteen consecutive patients with degenerative lumbar disease underwent eTLIF through paraspinal approach. Their clinical outcomes were evaluated with visual analog scale (VAS), Oswestry Disability Index (ODI) and the MacNab's criteria; radiological outcome measured with segmental lordosis (SLA), global lumbar lordosis (LLA), disc height (DH) on plain radiograph and percentage of potential fusion mass on CT scan at pre-operative, post-operative and final follow up period. intra operative and post-operative complications noted. Results: Mean age was 63. 71 years and Mean follow-up periods was 7.78 months. In the X-ray result, mean SLA improved in pre-operative/post-operative/ followup period 9.87 $\pm 2.740 / 11.79 \pm 3.740 / 10.56 \pm 3.690$ ( $p>0.01$ ); mean LLA improved 37.1 \pm 7.040 / $39.2 \pm 7.130 / 35.7 \pm 7.250$ ( $p>0.01)$. Mean DH improved from $8.97 \pm 1.49 \mathrm{~mm} / 12.34 \pm 1.39 \mathrm{~mm} / 11.44$ $\pm 1.98 \mathrm{~mm}(\mathrm{p}<0.01)$. In the CT result, Average percentage of fusion mass was $42.61 \%$. VAS was improved, 7.67/3.39/2.5 and ODI was improved, 74.9/34.56/27.76 by each preoperative/postoperative/final follow-up. In the clinical result, excellent was 5 cases and good was 13 cases. Conclusion: eTLIF was competent and viable surgical procedure. The results were excellent in the form of endplate preservation, disc height restoration, minimal post-operative pain with early mobilization. The fusion volume occupies $40 \%$ to $50 \%$ of disc space is expected to give sufficient fusion.

Key Words: Endoscopic TLIF, Paraspinal approach, Feasibility, End plate preservation

\section{INTRODUCTION}

Implementation of endoscopic spine surgery in the management of degenerative lumbar diseases has significantly reduced the need for fusion surgeries ${ }^{1,2)}$. This is in keeping with the philosophies of minimally invasive spine surgery as detailed by Young et al, where a staged approach to therapeutic interventions is recommended, beginning with the least invasive treatment options whilst reserving a fusion procedure as a last resort ${ }^{3,4}$. The lumbar fusion procedures are reserved for cases of spinal instability, spondylolisthesis and deformity where fusion surgery still stands as the first line of surgical management. The principles of minimally invasive surgery have also been applied to the lumbar fusion procedure, with the development of the minimally invasive transforaminal lumbar interbody fusion (MIS-TLIF). The performance of a MIS-TLF using an endoscope offers some distinct advantages, such as allowing better visualization the disc space, enhanced endplate preparation and contralateral decompression.

The objective of this study was to analyze the preliminary clinical and radiological outcomes of the recently developed technique of endoscopic transforaminal lumbar interbody fusion (eTLIF) performed through a paraspinal approach in patients with degenerative lumbar spine disease. 


\section{MATERIALS AND METHODS}

\section{Subjects}

This retrospective case study was approved by the institutional review board of Nanoori Hospital, Seoul, Republic of Korea. Informed consent was obtained from all patients who participated in study. We studied 18 consecutive patients with 20 levels of lumbar degenerative disease; ( 6 males, 12 females; Mean age 63.71 years) who underwent Endoscopic Transforaminal Lumbar Interbody Fusion (TLIF) through the posterior paraspinal approach at a specialized spine hospital between august 2018 and January 2019. Inclusion criteria were: degenerative grade 1 spondylolisthesis, lumbar central canal stenosis with foraminal stenosis and segmental instability confirmed on dynamic radiograph. Spondylolisthesis grade 2 and more and lumbar canal stenosis with more than $50 \%$ loss of disc height were excluded from this study.

\section{Surgical Procedure}

The procedure was performed under general anaesthesia. The patients were placed in a prone position on a radiolucent table over a Wilson's frame. The target/entry point is located under fluoroscopic guidance. The target/entry point is the ipsilateral facet at the level of the fusion and was approached through a conventional Wiltse's approach between the multifidus and longissimus muscles. Usually this interval is located approximately $3-4 \mathrm{~cm}$ lateral to the midline. A longitudinal skin incision of approximately $1 \mathrm{~cm}$ in length was made $4 \mathrm{~cm}$ lateral to the midline on affected side. This paraspinal approach facilitates the medial trajectory of the working cannula and cage into the disc space. Serial dilators were introduced through the interval and finally a working channel is introduced along with an endoscope. For the initial dissection we use a working cannula with an outer diameter of $13.7 \mathrm{~mm}$ and a bevelled tip. The endoscope has a 15 degree viewing angle, an outer diameter of $10 \mathrm{~mm}$, a working channel diameter of $6 \mathrm{~mm}$ and a working length $125 \mathrm{~mm}$. ILESSYSDelta $^{R}$ (Joimax $\mathrm{GmbH}$, Germany).

The soft tissue over the facet joint was dissected using a radiofrequency ablator to expose the inferior articular process (IAP) of the superior vertebra. An endoscopic drill is used to hollow out the groove at the IAP in an inverted "L" fashion. The IAP is harvested in total and is used as an autologus graft later on in the procedure. The underlying superior articular process (SAP) is burred out with the drill to expose the foraminal part of the deep layer of ligamentum flavum. Central and contralateral decompression can conveniently be achieved though the same interval by simply tilting the endoscope (Figure 1). Haemostasis and annulotomy are then performed with the use of the radiofrequency probe (Elliquence, New York, USA). The intervertebral disc space is subsequently cleared with the use of pituitaries. The most important step of the procedure is end plate prepara- tion. The advantage offered by endoscopic spine surgery is that meticulous end plate preparation can be performed under direct vision without damaging the end plates. End plate cartilage is removed with the use of a dissector and curettes to prepare the fusion bed. In order to protect the traversing nerve root, the tip of the bevelled cannula is rotated from a superior to a medial direction and inserted into the disc space through the annulo- tomy site. The autologus graft mixed with allograft is tamped into the disc space under fluoroscopic image guidance. A single large 3D printed cage (GENOSS, GS Medical, Republic of korea) packed with demineralised bone matrix (DBM) was inserted as obliquely as possible and the final position of the cage is checked both by fluoroscopy and directly using the endoscope. Finally, the working cannula was withdrawn, leaving a suction drain in situ (Picture 1).

After completion of the interbody fusion procedure, the construct was stabilized with the insertion of percutaneous pedicle screws. The incision used for the inetrbody fusion is also used for the insertion of the ipsilateral pedicle screws. The contralateral pedicle screws were inserted through 2 separate incisions. The rods were subsequently inserted after which compression in applied in a routine fashion. Depending on the bone quality, if required the pedicle screw fixation was augmented with bone cement.

\section{Outcome Evaluation}

\section{1) Clinical Evaluation}

Demographic data collected and all 18 patients were clinically evaluated on the basis of the Visual Analogue Scale (VAS) score
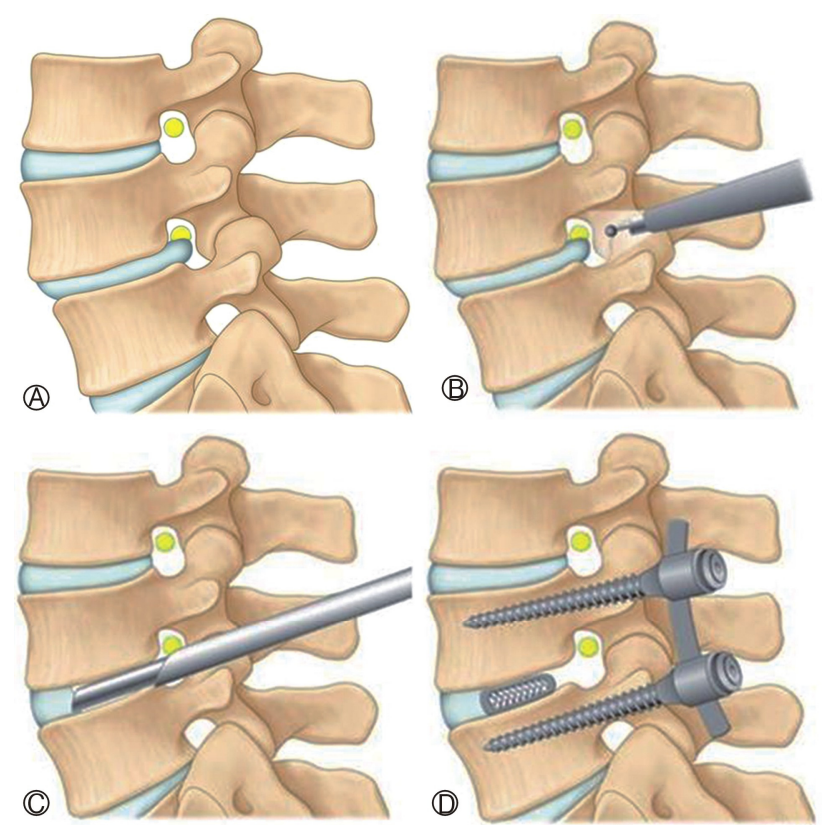

Figure 1. (A): Spondylolisthesis with foraminal stenosis, (B): Resection of superior and inferior articular process, (C): Discectomy and end plate preparation, (D): 3-D printed cage and percutaneous pedicle screw insertion. 
for the back, Oswestry Disability Index (ODI) and MacNab's criteria preoperatively, postoperatively and final follow up (minimum 6 months). Completeness of decompression was documented with postoperative magnetic resonance imaging (MRI). Patients were also assessed for any intra or postoperative complications and for recurrence of symptoms.

\section{2) Radiological Evaluation}

The radiological assessment was performed by an independent observer who was blinded to the patient's clinical information. Plain radiographs were used to evaluate disc height $(\mathrm{DH})$, lumbar lordosis angle (LLA) and segmental lordosis angle (SLA) pre and post operatively.

The fusion status was assessed using computed tomography (CT) at 6 months and 1 year following our CT protocol ${ }^{5)}$. The Bony fusion was defined when there is continuous contact of trabecular bone between upper and lower endplates of the fusion segments. Osteolysis at the margins of fusion devices (pedicle screws or cage), cystic changes within the endplates adjacent to the cage, linear defects in the trabecular bone bridge of fusion were considered features of delayed or failed fusion.

In order to evaluate the fusion mass, the CT scan was done with axial slices parallel to the end plates of the fused segment. The percentage of fusion mass surface area was calculated on CT images in axial cut sequences using software (INFINITT, INC, Seoul, Korea) in the following steps -

1) The surface area of fusion mass was measured at mid disc level from the region of interest (ROI). The surface area of cage was also included as we used 3D-printed cage with osteo-integration properties. The surface area of any osteophytes present at the end plates were excluded.

2) The surface area of upper and lower end plates measured from the CT slices going through or close to the end plates.

3) The average of the upper and lower end plate surface areas were calculated.

4) The percentage of fusion mass surface area was subsequently calculated using the formula below: percentage fusion=surface area of fusion mass/average end plate surface area $\times 100$ (Figure 2).

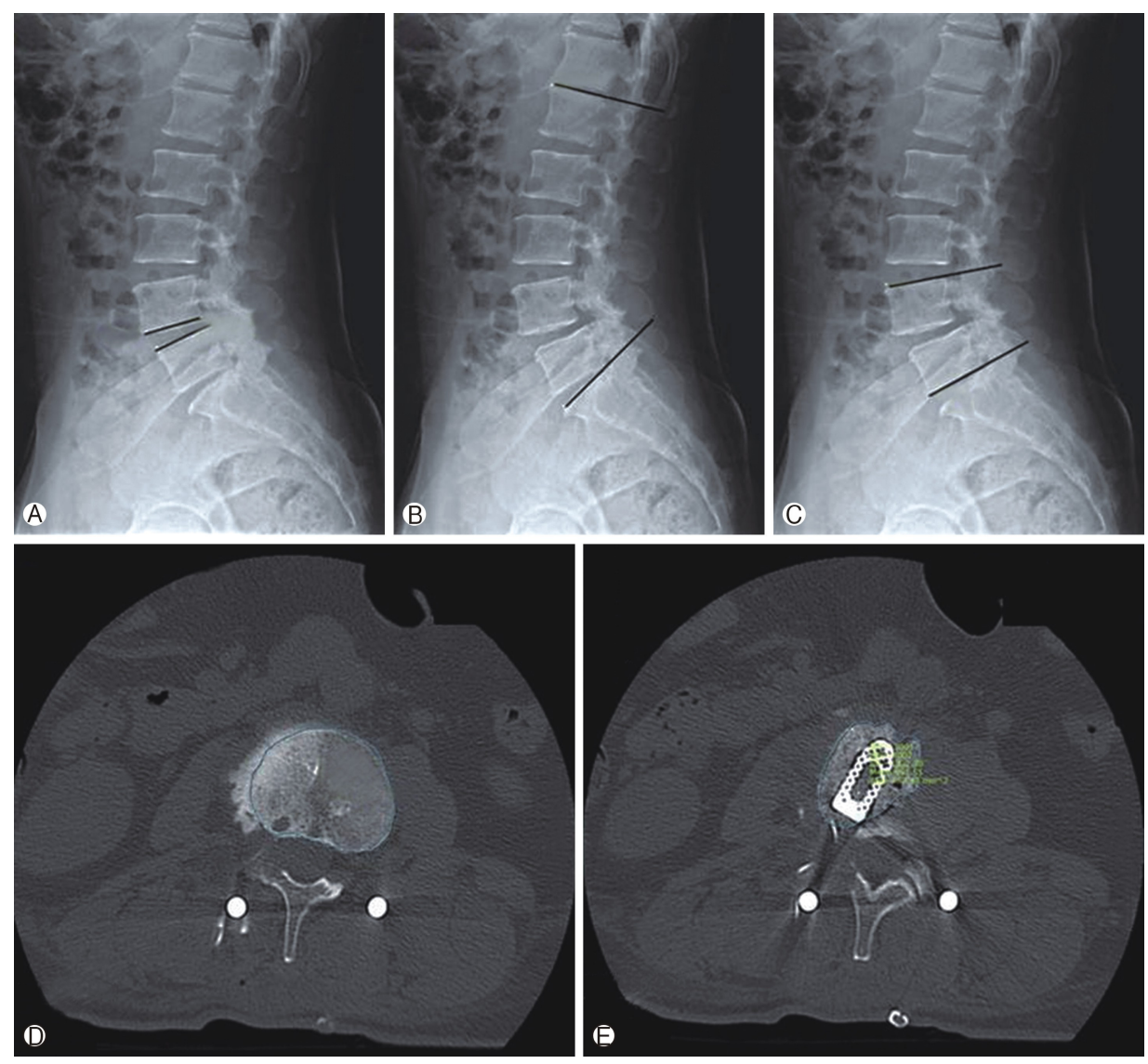

Figure 2. Radiological assessment. Plain radiograph used to evaluate, (A): Disc height (DH) distance between upper and lower end plate, (B): Lumbar lordosis angle (LLA) angle between superior end plate of L1 and S1vertebra, (C): Segmental lordosis angle (SLA) angle between superior end plate of upper and inferior end plate of lower vertebra of fused segment, (D): Measurement of surface area of upper and lower end plates on CT scan on axial slices going through or close to the end plates, (E): Measurement of surface area of fusion mass at mid disc level from the region of interest (ROI). 


\section{Statistical Analysis}

Clinical data was analyzed with SPSS version 18 statistical analysis software (IBM corporation, New York). The continuous variables were expressed as mean and standard deviation (SD). The paired t-test is used for comparison of preoperative and postoperative VAS, ODI scores; LLA, SLA and DH values. A value of $(p<0.01)$ considered significant.

\section{RESULTS}

\section{Clinical Outcomes}

Out of 18 patients, there were 10 patients with grade 1 spondylolisthesis, 4 patients with segmental instability, 3 patients with lumbar canal stenosis and 1 patient with lumbar disc herniation. 16 patients were operated for single level disease including L2-3 (1 patient), L3-4 (3 patients), L4-5 (11 patients) and L5-S1 (1 patient), while 2 patients were operated on for 2 level disease (one patient at L3-5 and one patient at L4-S1).

All patients were followed up for 6 months to 1 year with a mean follow-up period of 7.78 months. The mean VAS score significantly improved from preoperative levels on follow-up: $7.67 \pm 1.13 / 3.39 \pm 1.38 / 2.5 \pm 1.34(p<0.01)$. The mean ODI values also improved significantly: $74.9 \pm 8.03 / 34.56 \pm 8.80 / 27.76 \pm 8.64$ $(p<0.01)$. Based on MacNab's criteria, the clinical results were
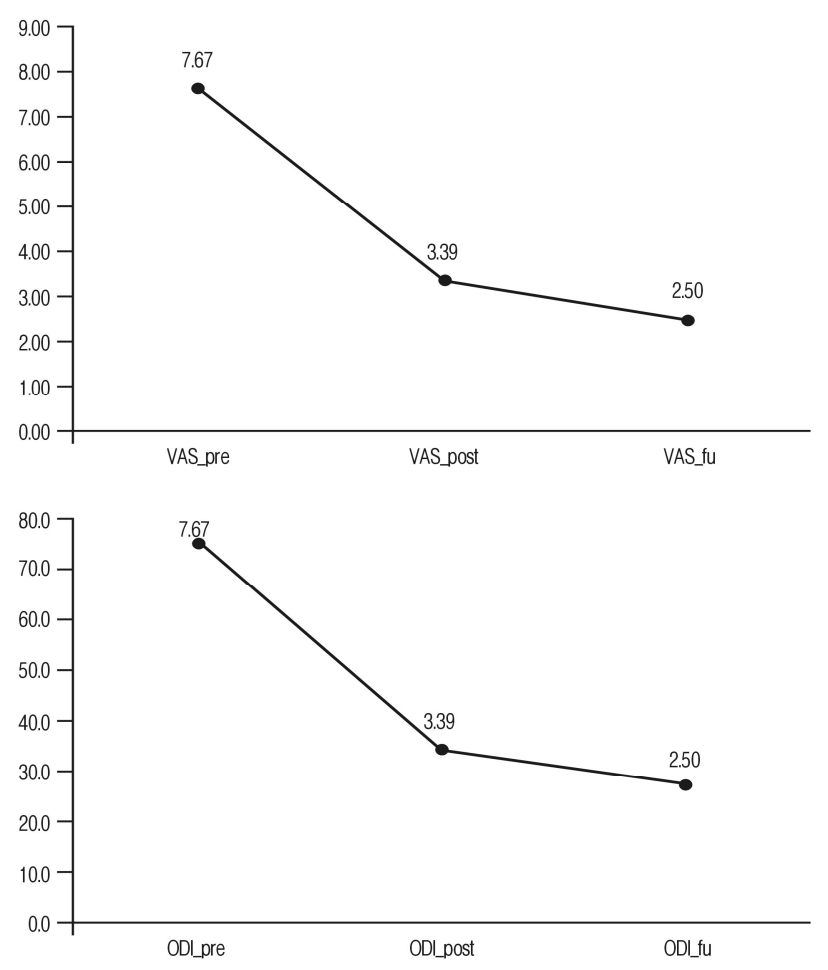

Figure 3. Patients pain assessment during pre-operative, post-operative and final follow up period. excellent in 5 patients and good in 13 patients (Figure 3).

\section{Radiological Outcomes}

In the X-ray result, mean preoperative segmental lordosis angle (SLA) $9.87+2.74$ degree changed to the final follow-up was $10.56+3.69$ degree $(p>0.01)$. Mean preoperative lumbar lordosis angle (LLA) was 37.1+7.04 degree and at the final follow-up was $35.7+7.25$ degree $(p>0.01)$. Mean preoperative disc height (DH) was $8.97+1.49 \mathrm{~mm}$ improved significantly to $11.44+1.98 \mathrm{~mm}$ $(p<0.01)$ at final follow up. When comparing the disc heights $(\mathrm{DH})$ between immediate post op to final follow up, there was a statistically significant difference $(p<0.01)$. The radiological outcomes using CT showed that the mean vertebral body end-plate average surface area was $1563.7 \mathrm{~mm}^{2}$ and average surface area of fusion mass was $663.7 \mathrm{~mm}^{2}$. The calculated average percentage of fusion mass was $42.61 \%$ (Table 1 ).

\section{Recurrence and Complications}

We recorded 2 intraoperative complications in the form of dural tears over the traversing nerve root sleeve which was managed conservatively using a fibrin sealant patch. Both the patients were asymptomatic in subsequent follow up and recovered well clinically.

We did not experience any postoperative complications in the form of a hematoma collection, infections, implant migration, or implant breakage. No patient required revision surgery.

\section{DISCUSSION}

Traditionally, open posterior lumbar interbody fusion (PLIF) and transforaminal lumbar interbody fusion (TLIF) have been established as the gold standard surgical treatments used in the management of degenerative lumbar spine conditions. However

Table 1. Patient's radiological data pre-operative, post-operative and final follow up period (values are presented as mean+standard deviation, $p<0.01$ considered as significant)

\begin{tabular}{l|r|l}
\hline \hline \multicolumn{1}{c|}{ Radiological Data } & value & $\mathrm{p}$-value \\
\hline Lumbar lordosis angle & & \\
pre operative & $37.1+7.040$ & \\
post operative & $39.2+7.136$ & $\mathrm{p}<0.01$ \\
final follow up & $35.7+7.252$ & \\
Segmental lordosis angle & & \\
pre operative & $9.87+2.743$ & \\
post operative & $11.79+3.742$ & $\mathrm{p}<0.01$ \\
final follow up & $10.56+3.690$ & \\
Disc height & & \\
pre operative & $8.97+1.490$ & \\
post operative & $12.34+1.399$ & $\mathrm{p}<0.01$ \\
final follow up & $11.44+1.986$ & $\mathrm{p}<0.01$ \\
\hline Mean percentage of surface area of fusion mass $42.61 \%$ & \\
\hline
\end{tabular}


it comes with unavoidable complications such as damage to the posterior ligamentous complex and paraspinal muscles which are associated with the development of failed back syndrome ${ }^{6}$. In 2003, foley et al, ${ }^{7)}$ developed the MIS-TLIF technique to overcome this problem using a $20-24 \mathrm{~mm}$ tubular retractor. However, with this technique it is difficult to observe the end plate during end plate preparation and it is relatively difficult to decompress the contralateral side. Lateral lumbar interbody fusion (LLIF) and Oblique lumbar interbody fusion (OLIF) have been developed to avoid injury to back muscles and improve visualization of the end plates ${ }^{8)}$, however these techniques are also associated with serious approach related complications such as potential injury to major vessels, ureter and lumbar plexus ${ }^{9,10)}$.

Successful clinical results in a fusion surgery depends upon achieving a high fusion rate along with maintenance of disc height and sagittal balance. Failure to achieve bony fusion may result in implant loosening, breakage and back pain in the long term. Previously reported fusion rates for PLIF ranged from 56 to $100 \%{ }^{11,12)}$; while fusion rate for open TLIF ranged from 86 to $100 \%{ }^{13,14)}$. Despite the narrow operative field in MIS-TLIF; several scientific papers have demonstrated good fusion rates ranging from $92-100 \%{ }^{15,16)}$.

Successful fusion primarily depends upon the surface area of the fusion bed and the correct placement of the bone graft along with the interbody cage over fusion bed. The inaccurate placement of bone graft over unprepared end plates with intact cartilage often results in pseudarthrosis. Potter et $\mathrm{al}^{13)}$ have reported that in order to obtain a firm interbody fusion, exposure of more than $30 \%$ of the endplate is required. With the transforaminal approach $56 \%$ of endplate can be prepared. Whilst performing endplate preparation, it is important not to remove excessive amounts of subchondral bone. Overenthusiastic disc preparation can result into subsidence and recurrent foraminal stenosis; particularly in osteoporotic patients. As we used a 15 degree endoscope inside the intervertebral disc space which can be rotated 360 degrees, it allows access to $60-80 \%$ of the end plate $^{17)}$. We calculated the mean percentage of surface area of fusion mass to be $42.61 \%$ which is more than sufficient to achieve interbody fusion. We used 3D-printed porous titanium interbody cages which provide an osteo-conductive scaffold for new bone growth. The cages provide short term stability due to friction and long term stability due to osteoblast adhesion, proliferation
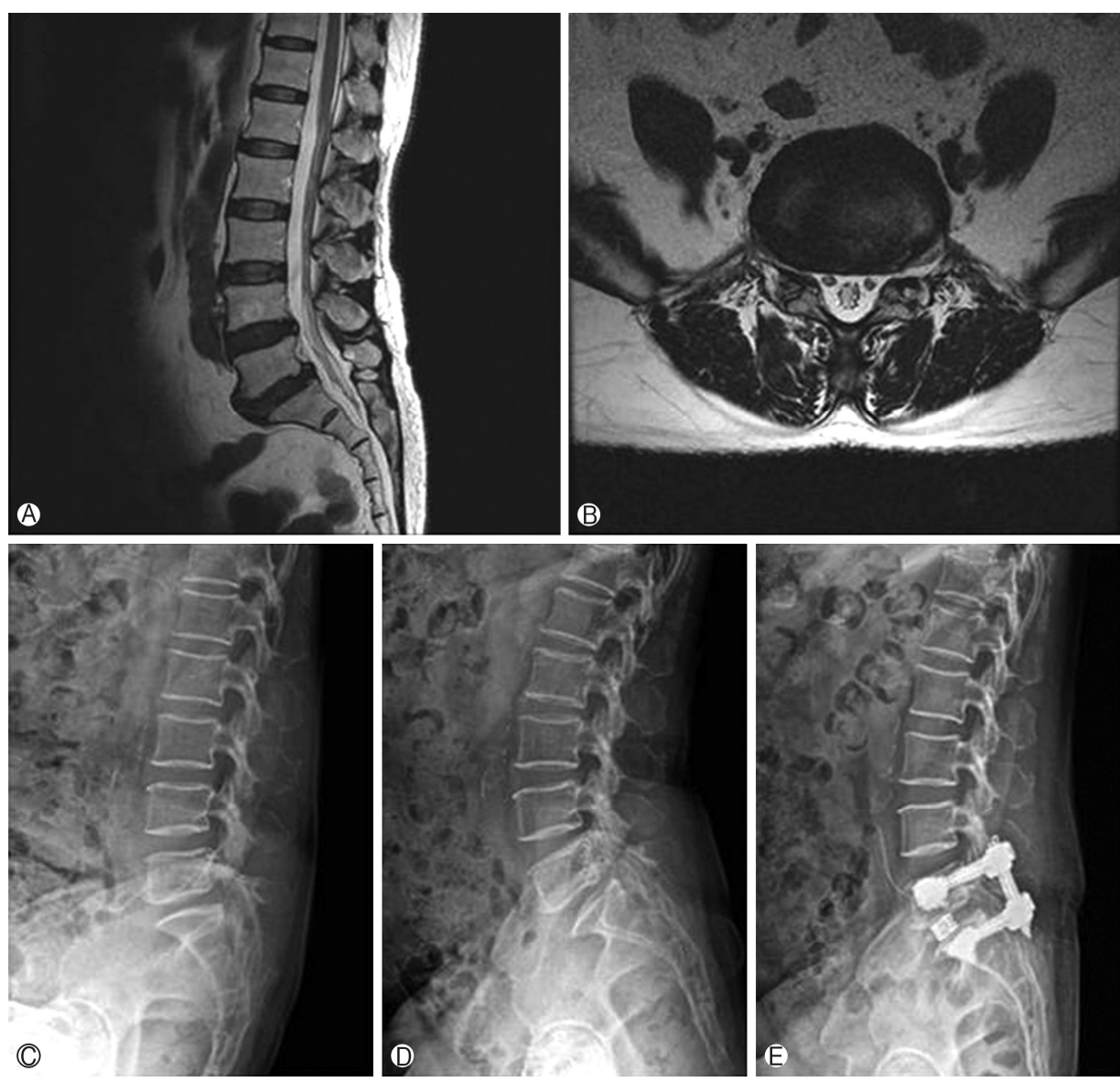

Figure 4. Representative case - 62 years female patient operated for L5-S1 endoscopic TLIF through paraspinal approach (A) \& (B) T2 weighted sagittal and axial image showing L5-S1 grade 1 spondylolisthesis with foraminal stenosis (C) \& (D) Dynamic radiograph showing angular instability E Post operative radiograph. 
and bone ingrowth. A single large cage improves the construct stability and bone ingrowth decreases subsidence due to load distribution ${ }^{18)}$. Fusion rates can be further enhanced with the use of rh-BMP and various other osteoinductive materials.

In 2012, Osman et al, ${ }^{19)}$ first reported the outcomes of 60 cases of endoscopic TLIF for the treatment of degenerative lumbar conditions with a $59.6 \%$ solid fusion rate with $36.2 \%$ of patients achieving a stable fixation. But the complication rate was upto $20 \%$. Recently, Mongesterns ${ }^{\text {it) }}$ et al, published the outcome in 30 patients operated with percutaneous TLIF with a mean follow-up period of 38 months. He reported a 100\% fusion rate using rigid PEEK cages and an expandable titanium cage. Lee et $\mathrm{al}_{1}{ }^{20)}$ performed percutaneous TLF in 18 patients using titanium expandable spacers (B-Twin) under local anesthesia with conscious sedation. He achieved good clinical results with $88 \%$ fusion rates (16 out of 18 patients) with an average subsidence of $2.1 \mathrm{~mm}$. Subsidence of the interbody cage with a loss of interbody disc height is expected in the post-operative period; however progressive subsidence can cause a reduction in foraminal height which results in recurrent foraminal stenosis and loss of sagittal balance
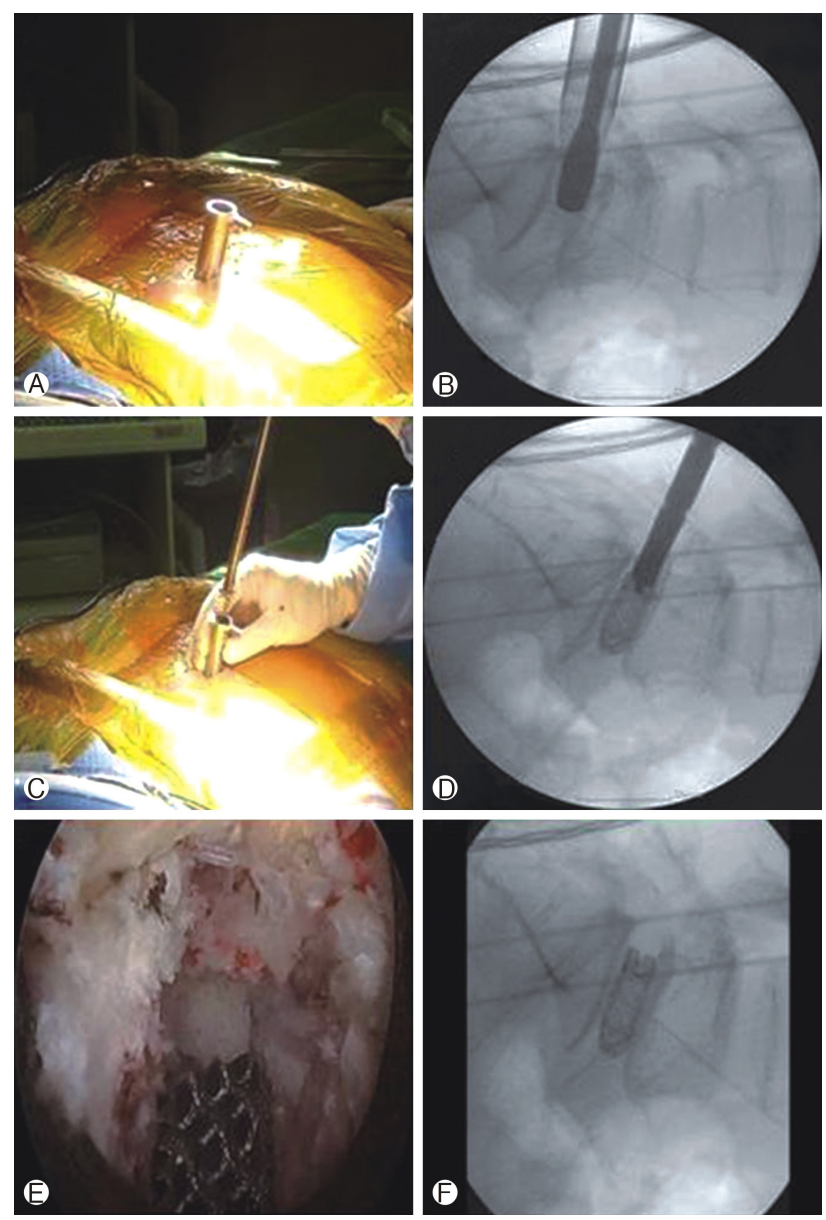

Figure 5. (A) \& (B) beveled working cannula inserted with its tip inside disc space and end plate preparation done (C) \& (D) 3-D printed cage inserted through obturator and then it is removed (E) \& ( $($ ) Final position of implant checked under endoscopic view and fluoroscopy guidance. also. In the present study we did not find any radiological signs of non-union, but we did experience a subsidence rate of $0.9 \mathrm{~mm}$. There was a significant difference between pre-operative measurements and measurements at final follow-up $(p<0.01)$. Significant subsidence is defined as a decrease in disc height of more than $3 \mathrm{~mm}$. Although our subsidence rate was low; it does require close long term follow up.

Jacquet et $\mathrm{al}_{1}{ }^{21)}$ considered the procedure controversial due to the technical difficulty and reported high complication rates (up to 36\%). They also emphasized the need for technical improvements to the procedure. Most studies describe the utilization of the classical endoscopic transforaminal approach with expansile foraminoplasty for disc space preparation and cage insertion. Majority of these studies describe the development of the common postoperative complication of dysaesthesia due to irritation of DRG. This usually occurs whilst performing disc preparation and/or during the insertion of a large cage into the disc space. In our study we utilized a conventional Wiltse's approach which minimizes the need for retraction of the exiting root and DRG and thus we did not see this complication of post-operative dysaesthesia in our study population. However, we did have a complication rate of 3.6\% secondary to dural tears in 2 patients which occurred over traversing root sleeve. These injuries occurred secondary to incomplete retraction of traversing root by the bevel tip. This complication can be minimized by carefully rotating the bevel tip and introducing it into the disc space, thereby safely retracting the traversing nerve root during disc space preparation and cage insertion.

Interbody fusion via the transforaminal approach has certain limitations such as inadequate foraminoplasty which could make cage insertion difficult, and also the iliac crest can act as an obstacle for the insertion of an interbody cage into the L5-S1 disc space. Such difficulties can often be overcome using the paraspinal approach. Despite the encouraging clinical results, endoscopic TLIF has certain limitations which needs further technical development; it is not suitable for grade 2 or more spondylolisthesis due to difficulty to achieve complete reduction and sagittal balance. In patients where the disc space is collapsed more than $50 \%$, it is very difficult to introduce the endoscope into disc space. Osman et $\mathrm{al}^{177}$ has advised prior pedicle screw instrumentation and distraction of the disc space prior to introduction of the endoscope to counter this problem. In our study we did not find a statistically significant difference in segmental balance from pre-operation to final follow-up. Although the technique of endoscopic TLIF is similar to open TLIF, its ability to restore segmental balance cannot be confirmed in the present study. Further technical developments in instrumentation may be required to achieve disc space distraction, and also in cage design perhaps with the use of expandable or hyperlordotic cages to help restore segmental balance. Lewandrowski et $\mathrm{al}_{1}{ }^{24}$ performed endoscopic TLIF with a standalone lordotic cage for a single level with good clinical results (77.8\%). However, he reported cage subsidence in all the cases with revision surgery rate of around $8 \%$ (4 out of 48). Long term radiological studies with respect to subsidence rate, sagittal balance, effect at adjacent segment, fusion mass distribution and fusion rate will be helpful for future develop- 
ment of endoscopic instrumentation for fusion surgery. Certain studies have also proposed the feasibility of using biportal endoscopic surgery for interbody fusion with encouraging clinical results ${ }^{22,233}$.

This is a retrospective study with a small sample size providing preliminary results. We ideally need to compare an Endoscopic TLF group with a matched control group like an open or MIS TUF group in order to more accurately compare the effectiveness of Endoscopic TUF compared with existing lumbar fusion techniques. A prospective study with large sample sizes will give more com- prehensive results.

\section{CONCLUSION}

According to the results of this study, endoscopic TLIF was feasible procedure with an advantage of better visualization and preservation of endplate, minimal blood loss and minimal postoperative pain with early mobilization. In addition, the fusion volume as measured on post-operative CT scans, occupied $40 \%$ to $50 \%$ of disc space along with the use of 3D printed cages expected to give higher fusion rates. In conclusion, our preliminary results show that endoscopic TLF is considered to be a viable surgical procedure with further long-term follow-up warranted.

\section{CONFLICT OF INTEREST}

Dr. HS Kim is an editorial board member of the journal but was not involved in the peer reviewer selection, evaluation, or decision process of this article. There are no other potential conflicts of interest relevant to this article to declare.

\section{REFERENCES}

1. Kim M, Kim HS, Jang IT, et al.: Evolution of Spinal Endoscopic Surgery. Neurospine 16(1):6-14, 2019 doi:10.14245/ns.1836322. 161.

2. Wang JC, Kim HS: Endoscopic Spinal Surgery (ESS): Prepare for a Happy 100-Year-Old!. Neurospine Mar;16(1):4-5, 2019

3. Yeung A, Kotheeranurak V. Transforaminal Endoscopic Decompression of the Lumbar Spine for Stable Isthmic Spondylolisthesis as the Least Invasive Surgical Treatment Using the YESS Surgery Technique. Int J Spine Surg Jun;12(3):408-414, 2018

4. Gore S, Yeung A: The "inside out" transforaminal technique to treat lumbar spinal pain in an awake and aware patient under local anesthesia: Results and a review of the literature. International Journal of Spine Surgery 8, 2014.

5. Williams AL, Gornet MF, Burkus JK: CT evaluation of lumbar inter body fusion: Current concepts. American Journal of Neuroradio logy 26(8)2057-2066, 2005

6. Kawaguchi Y, Matsui H, Tsuji H: Back muscle injury after posteriorlumbar spine surgery. Part 1: Histologic and histochemical analyses inrats. Spine 19:2590-2597, 1994

7. Foley KT, Holly LT, Schwender JD: Minimally invasive lumbarfusion. Spine (Phila Pa 1976). 28(15 Suppl):S26-35, 2003

8. Anand N, Rosemann R, Khalsa B, Baron EM: Mid-term and longterm clinical and functional outcomes of minimally invasive correction and fusion for adults with scoliosis. Neurosurg Focus 28: E6, 2010

9. Walker CT, Farber SH, Cole TS, et al.: Complications for mini- mally invasive lateral interbody arthrodesis: A systematic review and meta-analysis comparing prepsoas and transpsoas approaches. J Neurosurg Spine Jan;25:1-15, 2019

10. Joseph JR, Smith BW, La Marca F, Park P: Comparison of complication rates of minimally invasive transforaminal lumbar interbody fusion and lateral lumbar interbody fusion: A systematic review of the literature. Neurosurg Focus Oct;39(4):E4, 2015

11. Brantigan JW, Steffee AD, Lewis ML, Quinn LM, Persenaire JM: Lumbar interbody fusion using the Brantigan $\mathrm{I} / \mathrm{F}$ cage for posterior lumbar interbody fusion and the variable pedicle screw placement system: Two-year results from a Food and Drug Administration investigational device exemption clinical trial. Spine (Phila Pa 1976) 25:1437-1446, 2000

12. Kai Y, Oyama M, Morooka M: Posterior lumbar interbody fusion using local facet joint autograft and pedicle screw fixation. Spine (Phila Pa 1976) 29:41-46, 2004

13. Potter BK, et al.: Transforaminal lumbar interbody fusion: Clinical and radiographic results and complications in 100 consecutive patients. J Spinal Disord Tech 18:337-346, 2005

14. Hackenberg L, et al.: Transforaminal lumbar interbody fusion: A safe technique with satisfactory three to five year results. Eur Spine J 14:551-558, 2005

15. Schwender JD, Holly LT, Rouben DP, Foley KT: Minimally invasive transforaminal lumbar interbody fusion (TLIF): technical feasibility and initial results. J Spinal Disord Tech 18 Suppl: S1-S6, 2005

16. Kim MC, Chung HT, Kim DJ, Kim SH, Jeon SH: The clinical and radiological outcomes of minimally invasive transforaminal lumbar interbody single level fusion. Asian Spine J 5:111-116,

17. R Morgenstern and C Morgenstern: "Percutaneous Transforaminal lumbar interbody fusion (pTLIF) with a posterolateral approach for the treatment of denegerative disk disease: Feasibility and preliminary results," International Journal of Spine Surgery 27(9):41, 2015

18. McGilvray KC, Easley J, Seim HB, et al.: Bony ingrowth potential of 3D-printed porous titanium alloy: A direct comparison of interbody cage materials in an in vivo ovine lumbar fusion model. Spine J 18(7):1250-1260, 2018

19. SG Osman: "Endoscopic transforaminal decompression, interbody fusion, and percutaneous pedicle screw implantation of the lumbar spine: A case series report," International Journal of Spine Surgery 6(1):157-166, 2012

20. SH Lee, HY Erken, J Bae: "Percutaneous transforaminal endoscopic lumbar interbody fusion: Clinical and radiological results of mean 46-month follow-up," BioMed Research International 2017

21. F Jacquot and D Gastambide: "Percutaneous endoscopic transforaminal lumbar interbody fusion: Is it worth it?" International Orthopaedics 37(8):1507-1510, 2013

22. Hwa Eum J, Hwa Heo D, Son SK, Park CK: Percutaneous biportal endoscopic decompression for lumbar spinal stenosis: A technical note and preliminary clinical results. J Neurosurg Spine Apr; 24(4):602-607, 2016

23. Kim JE, Choi DJ: Biportal Endoscopic Transforaminal Lumbar Interbody Fusion with Arthroscopy. Cin Orthop Surg 10(2):248252, 2018

24. Lewandrowski KU, Ransom NA, Ramírez León JF, Yeung A: The Concept for A Standalone Lordotic Endoscopic Wedge Lumbar Interbody Fusion: The LEW-LIF. Neurospine Mar;16(1):82-95, 2019 